essential lines of attack on problems of disease, that cannot perhaps be solved without the additional aid of the laboratory and animal experiment. I cannot believe that any medical research worker can afford to divorce himself from clinical studies. When he does so he invariably loses touch with reality.

\section{External Dangers}

If the shortcomings of much medical research that $I$ have indicated really obtain, they are such as can be remedied only by those engaged in that research. Yet there are external dangers that increasingly surround research as it becomes progressively more organized from without by controlling bodies whose paid workers the researchers are. I have ventured to suggest that many more such workers are ready to make observations or experiments than appreciate the necessity for continued thought about their work and its implications. This tendency is not checked when research organizations expect their workers to manifest productivity in terms of published papers. The man who has every year to draw up his annual report showing what he has accomplished can scarcely be blamed if he does not spend much time in contemplation. $\mathrm{He}$ will not care to report that he has as yet published nothing, is not ready to publish anything, and has not got very much further than surveying his problem.

Some sacrifices of independence and freedom have to be made when research is widely organized and research workers are paid salaries. Even research foundations have to satisfy their "public" that they are getting results; they have to do some window-dressing. Only an informed and enlightened control can secure for the research worker that freedom from which alone his best work can flow. He must have time to think, and be encouraged to do so, and he must not be haunted by the fear that his productivity is being measured in terms of papers or that his share of the organization's financial resources is grudged him. Under the experienced and sympathetic administration of the more important research foundations he is least likely to suffer in this way, but if and when the State or public bodies-through hospital and other services-take a hand in the matter, and if considerations of political expediency begin to appear, the salaried research worker may end by becoming a kind of scientific helot from whom quick results, in directions not always determined by him, are looked for. Now is the time to safeguard him from this melancholy fate.

One last danger should, it seems to me, be foreseen in the organization of medical research in the new order-namely, the danger that the worker in pure research may be segregated in central institutes remote from hospitals and medical schools. I believe this would be fatal to true progress in medicine. It would take the spirit and the inspiration of inquiry from the schools, wherein alone are to be found the recruits to the ranks of original investigators. It would deprive the student of contact with the research worker, and would deaden the quality of the endeavour of everyone concerned in hospital work and medical teaching. The research worker himself would suffer no less from this isolation from the world of medical endeavour, from the true battle-front of medicine. Thus isolated he would soon lose his sense of reality and of proportion. He would cease to have the necessary vivid awareness of the nature and difficulties of medical problems. It has commonly been so in the past.

\section{Conclusion}

It is idle to look back, but how enviable in retrospect was the lot of the physician or surgeon of a past generation who engaged in research work to satisfy his own scientific curiosity! He could think at his leisure, could publish his results when these were ripe and at his chosen moment: he was not harassed by the thought that something was expected from him at stated intervals in the calendar, or by the fear that some organization would begin to think that he was not worth his keep as a research worker. It may be true that we can no longer leave the matter of medical research to his amateur care; but will the subsidized research worker, caught up in the machine of organized research, ever savour the true taste of discovery so fully? It is hard to believe that the human contacts the older workers made in the practice of medicine were not at least one source of their inspiration, or that the time they gave to the treatment of the sick was really the waste some modern advocates of reform would have us think.
Therefore, while fully recognizing that the times before us demand a more organized research effort than we have had hitherto, we may yet regret the probability that something of what was most favourable to the best work may be lost in the process, and we may hope that those who are reorganizing the fabric of British medicine will bear all this in mind and will make the conditions under which the salaried research worker has to labour and live most favourable to the free and unhampered development of his powers.

\section{THE PATHOLOGY OF CLOSED INJURIES OF THE CHEST* \\ BY}

J. V. WILSON, M.D.

Major, R.A.M.C.; D.A.D.P. of a Command

After two years of continuous war work I shall here attempt to review in broad detail some experiences gained in the routine post-mortem examination of battle casualties, selecting for special analysis thoracic injuries of the closed type. My material has been drawn from both Service and civilian casualties, and it can be claimed to be wide and varied.

By a closed injury to the chest I mean either that the skin and subcutaneous tissue were intact or that, if these were pierced, the course of the missile had been such as to leave the pleura intact and so the pleural space had no contact with the outside air. This study excludes all cases in which a foreign body had entered the pleura or thoracic viscera, and deals with a group of cases that, to my mind, are the most interesting and in someways the most important of war injuries. The cases may be divided into two main categories-those due to direct violence to the chest and those in which the injury to the thoracic viscera is indirect-i.e., a result of injury elsewhere in the body. Fat embolism is an example of this latter group: here the lung changes are very definite, but are usually the result of injury elsewhere, such as fracture of long bones. The former group includes: (a) lacerations, $(b)$ contusions, $(c)$ blast injuries (pulmonary concussion); it is principally with these cases that I shall deal.

\section{Lacerations}

Laceration of the heart or great vessels is of little pathological interest. It rarely if ever occurs in closed chest injuries, and when it results in rupture is rapidly fatal. A case of ruptured aorta due to blast has recently been reported by Wilson and Tunbridge, but $I$ have not seen any further case of laceration of heart or large vessels in my series of war injuries. Lacerâtion of the lung, on the other hand, is of great importance, and has occurred in this series with considerable frequency.

Case 1.-A gunner aged 28 was admitted suffering from shock and complaining of pain in the right chest. A bomb had exploded near his gun -position and he had been thrown up against part of the gun. It was not clear whether he had been actually struck by a bomb splinter: the only evidence of external violence was a cut on the forehead about 2 in. long just to the right of the midline and extending upwards from the root of the nose. He was tender all over his right chest and blood was present in the right pleural cavity. Radiographs revealed multiple fractures of the ribs and a large right-sided pleural effusion. He died about six hours after admission.

At the post-mortem examination there was no evidence of external injury to the chest. The $3 \mathrm{rd}$ to 10 th ribs on the right side were fractured just beyond the neck, while the 6th, 7th, and 8th ribs were also fractured in the mid-axillary line. The right pleural space contained about $1,000 \mathrm{c.cm}$. of fluid blood and a small blood clot. The right lung was lacerated by the fractured ribs along the line of the angle of the ribs, there were multiple areas of haemorrhage all over its substance, and it was partially collapsed, a deposit of fibrin being present on the diaphragmatic surface and the lower half of the lower lobe. The left pleural space was intact but contained a small excess of serous fluid, the lung itself being congested but with no definite localized areas of haemorrhage. The gunner had been standing about 40 yards away when the bomb

* The opening address at a B.M.A. meeting (Over-seas Branch), April 30, 1942. 
exploded, and most of his injuries would appear to have been due to direct violence, either from a flat bomb splinter or from being thrown against a solid object.

Case 2-An officer was working with a party of men on an aerodrome when a bomb exploded $150 \mathrm{ft}$. away. He was hit on the right side by a flat piece of bomb splinter and thrown to the ground. There were no external injuries except bruising of the right upper arm and right chest wall. On admission he was shocked and collapsed, and died about an hour later.

Necropsy revealed no external injury to the chest wall, but there was considerable bruising of and haemorrhage into the muscles of the right chest wall, especially posteriorly. The 4th, 5th, and 6th ribs had been fractured posteriorly along the angle of the ribs and again in the anterior axillary line. They had been driven in, rupturing the parietal pleura and intercostal vessels. The right pleural space contained a small amount of air and $4,000 \mathrm{c} . \mathrm{cm}$. of fluid blood-i.e., a pneumohaemothorax had developed. There was no collapse of the right lung, but the mediastinum had been pushed across to the left side. The right lung was the seat of a large haemorrhage which involved the lower half of the upper lobe, the middle and lower lobes except their anterior margin, and extended throughout the substance of the lung. Three large subpleural bullae were present-one on the anterior aspect of the lower part of the upper lobe, one in the interlobar surface of the middle lobe, and a third, which had ruptured, on the posterior aspect of the lower lobe. This lung had been torn superficially on the posterior aspect opposite the angles of the broken 4th, 5th, and 6 th ribs. The left lung was congested throughout and there was a haemorrhagic area in the posterior portion of the lower lobe, involving the deeper portions as well as the subpleural area.

These cases illustrate the chief features of lung laceration in closed chest injuries, and it will be realized that their seriousness depends not so much on the actual lung injury as on the development of complications. Laceration of the lung is rarely severe and seldom causes death in itself: a haematoma forms round the actual tear, but, owing to collapse of the lung tissue, haemorrhage soon stops. The neighbouring lung shows congestion, but there is seldom any evidence of gangrene of the torn edges, since the blood supply is so generous.

\section{Complications}

The complications which occur and which may prove so dangerous may be grouped as follows: (1) pneumohaemothorax ; (2) compression or collapse of lung ; (3) infection.

1. Pneumohaemothorax.-The degree of pneumothorax present is as a rule very slight. The clot which forms usually seals off the alveoli, and collapse of the surrounding lung helps this process, so that the amount of escaping air is small and would appear to be quickly absorbed. Subpleural bullae are rarely extensive and seldom rupture. It is true that the nature of the lung injury may be such that a valve may be formed and in this way a tension pneumothorax may develop, but in my experience this is extremely rare. Haemothorax, on the other hand, is common, and may be very extensive. There are two possible sources of origin for blood in the pleural space ; it may result from:

(a) Bleeding from the intercostal and mammary vessels, which, being systemic, may be very profuse and difficult to stop. (b) Bleeding from the pulmonary vessels at the site of laceration. This is rarely severe or fatal in itself. The pressure in the pulmonary vessels is low, the mean arterial pressure in the pulmonary circuit being about one-sixth that of the mean arterial pressure in the aorta, while the pressure in the pulmonary veins is near the zero level. The surrounding lung tends to collapse and the diaphragm on the injured side rises, so that Nature can in most cases be left to deal with this type of bleeding.

The differentiation of these two varieties of injury is important from the point of view of lung surgery.

The pleura is a mesothelial structure and reacts to haemorrhage as if it were a foreign body-i.e., with a mild inflammatory type of reaction. Locally there is an outpouring into the pleural space of a fluid rich in protein, accompanied by fever that is constant and without any evidence of toxaemia and so differs from the swinging temperature in the toxic patient with empyema. The local exudate of fluid not only increases the volume of the haemothorax present but may also cause a mild dehydration, which must be considered in treatment. Its presence and extent can be gauged fairly accurately by com- paring the haemoglobin content of the pleural fluid with that of the patient's blood. An interesting fact about haemothorax is that the blood seldom clots to any great extent. A small clot may be present, but the bulk of the blood is still fluid. This may result from either of two factors-it may be due to the fact that there is little tissue destruction and so thrombokinase is absent and hence clotting is greatly delayed, takes place only partially, or does not occur at all ; or it may be that clotting does not take place because, by the action of the lung in expanding and contracting, the blood is defibrinated and so in effect cannot clot. From examinations of large numbers of cases it would appear that no single hypothesis can fit every case; but studies of the calcium content and the clotting and prothrombin times of the blood from the haemothorax show that both the above factors play a part, and which of them predominates would seem to depend on the nature of the injury. Often tissue destruction (as in the cases cited) is considerable, ribs may be fractured, and the muscles and the parietal pleura torn, yet the blood may be still fluid. Fibrin in large amounts may be present on the lung surface, and thus defibrination may have taken place. In other cases the tissue destruction may be small and the absence of thrombokinase may be the deciding influence.

It has been stated that the fibrin deposited on the pleura may organize and so prevent the lung re-expanding. This would seem possible in cases in which lung collapse is of some duration, but I have never met with it in this series.

2. Compression or Collapse of Lung.-Compression of the lung may be acute as in pneumothorax or may come on gradually as in haemothorax. These cases of haemothorax may not reach clinical significance until 12 to 18 hours, but may finally attain enormous proportions, even as much as 3,000 to $5,000 \mathrm{c.cm}$. Here the heart is pushed over to the opposite side and the circulation and aeration of the opposite lung are severely impaired. Massive collapse is a rare occurrence; but its possibility should be kept in mind, as early diagnosis is of importance. It would appear to be due to loss of the cough reflex by shock or pain, so allowing mucus to collect and finally block a bronchus, or else a blood clot may find its way from the site of lung injury and likewise cause blockage. When this type of collapse is only temporary no definite pathology may be found in the lungs and complete recovery may take place, but infection or even bronchiectasis is always a possibility if the condition is of any standing.

3. Infection.-Even with the introduction of the sulphonamides infection is still the dread of the lung surgeon. In closed injuries of the chest it is rare for the pleura to be infected until later in the course, but pneumonic changes may set in early. They have no special pathological features.

\section{Contusions}

This group comprises injuries due to direct violence; these occur chiefly in younger persons whose ribs are still elastic, and so the underlying structures, heart, and lungs can be contused without actual external injury or fracture of the ribs themselves. I also place in this category an indefinite type of lung injury with an intact thorax which has been reviewed by Fallon (1940). He described the case of a medical student who was knocked down when boxing and later developed a cavity in the left lower lobe with a fluid level, a condition which resolved sporitaneously and resulted in full recovery. Here the pathology is indefinite, but in this series of war injuries the basic lesion is more easily recognized.

\section{(a) Cardiac Contusion}

That there can be contusion of the myocardium without any evidence of external injury or fracture of the ribs or sternum has been known for some time, and Barber $(1938,1940)$ has reviewed the effects of trauma on the heart and discussed the significance of myocardial contusion. He concludes that, while it is a definite pathological entity, the clinical diagnosis is difficult and "more cases of heart disability following trauma are overlooked than are diagnosed by mistake." Anderson (1940) has reviewed the literature on non-penetrating injuries to the heart and reported a new case. He states that injuries to the heart by nonpenetrating blows are commoner than has been supposed, and 
discusses the pathological and clinical picture. Warburg (1938), in his classical monograph, reviews 202 cases of traumatic injury to the heart. Bright and Beck (1935) have also contributed to the literature, while Moritz and Atkins (1938) have made an interesting experimental and pathological study of cardiac contusion. The importance of the subject is thus now generally recognized, and in all cases in which the history may point to injury to the chest wall evidence of cardiac trauma should be sought for clinically and the possibility of a cardiac element in the general picture eliminated. The whole condition may of course be subclinical, and complete recovery may take place. When it is at all severe we get evidence of myocardial damage such as cardiac irregularities or the development of angina pectoris, valvular lesions, or pericarditis.

In my war experience traumatic cardiac lesions have occurred in two definite circumstances-either from direct trauma to the chest or as a feature of blast injury. Owing to the nature of blast and the difficulty of getting an accurate story of the complete incident it is often impossible, when a bomb explodes, to say that the victim is not either hit directly or thrown against a firm structure, suffering direct violence to the chest. Since the difficulty of differentiating the cause exists, and since the subsequent pathological picture of the cardiac lesion is similar, I shall deal with both groups together. Trauma of the heart may cause contusion of the myocardium, traumatic pericarditis, or rupture of the valves and great vessels. Those subjected to war injuries are as a rule healthy persons, and so previous cardiac lesions do not complicate the picture.

Myocardial Contusion.-If we examine a transverse section of the thorax and note the position of the heart in relation to the spinal column behind and the sternum in front we can see how it is possible, especially in young subjects with elastic ribs, for the heart to be damaged without injury to the chest wall itself. Any part of the myocardium may be involved, but it would seem that the anterior surface of the ventricles was most often affected. At necropsy the pericardial fluid may be blood-stained and increased in amount. The myocardium at the site of contusion is dark and haemorrhagic, and has a nakedeye appearance not unlike an infarct. Microscopically, in recent cases there are subpericardial haemorrhages and evidence of tissue disorganization and generalized congestion. If the lesion is extensive and involves a considerable thickness of muscle the softening which occurs before organization may be serious enough to cause rupture of the myocardium as in the case reported by Priest in 1939 . Older cases in which organization has taken place are particularly difficult to differentiate from an infarct. The history, the site of the lesion, and the state of the coronary vessels must be taken into account.

Pericarditis.-Traumatic pericarditis is rarely serious, though Warburg (1938) found a purulent pericarditis in five cases of his series, and described a case of Pick's disease occurring two years after an injury. As most of our cases of cardiac injury due either to direct violence or to blast resulted in death soon after the injury, pericarditis was not seen.

Valvular Lesions and Rupture of Great Vessels.-Kahn and Kahn (1928) have shown, by striking the chest of a corpse, that it is possible to rupture a valve and, though I have never actually succeeded in doing this, several workers have recorded similar results. Moritz and Atkins (1938) in their experimental work report no rupture of valves in any of the 32 dogs whose hearts were subjected to trauma. Elsewhere I have recorded a case of ruptured aorta and tearing of an aortic cusp as a result of blast, but this lesion would appear to be rare.

\section{(b) Pulmonary Contusion}

In these days of blast, when the proper interpretation of the lesions incidental to bomb explosions is so essential, cases of pure pulmonary contusion are very important, and so a description of one may be of interest.

A soldier aged 26 was admitted to hospital, having been shot through the left shoulder with a rifle bullet fired at a distance of approximately 20 yards. The bullet passed over the neck of the humerus, hit the clavicle, fracturing it in several places, and had apparently been deflected up into the neck, passing behind the cervical vertebrae and emerging from the neck posteriorly on the right side. The patient died 6 hours later owing to shock and haemorrhage from the neck wound. The upper four ribs had been driven in without being fractured, and at necropsy the apex of the left upper lobe was contused, the pleura being intact. There was about $100 \mathrm{c.cm}$. of blood-stained fluid in the left pleural space. The left lung was congested throughout and the apex was the seat of a large area of haemorrhage situated on the lateral and anterior surface-e.g., peripheral rather than central. Anteriorly its border was very irregular and showed linear markings corresponding to the 2nd and 3rd ribs. The right lung was also congested throughout, but no definite areas of haemorrhage were present. There were also suggestions of linear markings on this side.

Histological examination of the left apex (the site of the contusion) showed alveoli packed with red blood cells. The capillaries were congested and a few were ruptured. The alveoli were intact and there was no evidence of oedema. The main feature of the left lower lobe was slight generalized congestion, but the whole of the right lung showed scattered areas of haemorrhages into the alveoli and generalized capillary congestion. Slight oedema was present and some of the alveoli were ruptured.

The interpretation of this case would seem to be that direct trauma affected the left upper lobe, causing haemorrhage throughout that lobe. The left lower lobe apparently escaped, but the right lung suffered contrecoup injuries, which produced naked-eye and microscopical pictures identical with those found in pulmonary concussion. It would thus appear that direct injury can produce all the essentials of a blast injury-an observation the importance of which will be discussed later.

\section{Blast Injuries}

Blast injuries, especially as they affect the lung (pulmonary concussion), have provided one of the most interesting studies in this Command during the present war.' The weight of the aerial attack and the structure of many of the shelters made its incidence high. I have had the opportunity of examining a number of large and small series of cases, as well as many individual ones, and so have been able to get some idea of their basic pathology and usual clinical course.

In blast the lung picture is usually one of haemorrhage. These areas of haemorrhage are scattered throughout the whole of the organ, and are found deep in the lung substance as well as being subpleural. There is no suggestion of consolidation, and the general feel of the lung is rubbery. Dark linear markings are present in adults just as often as in children, and from a study of the lung position in some of our later cases I

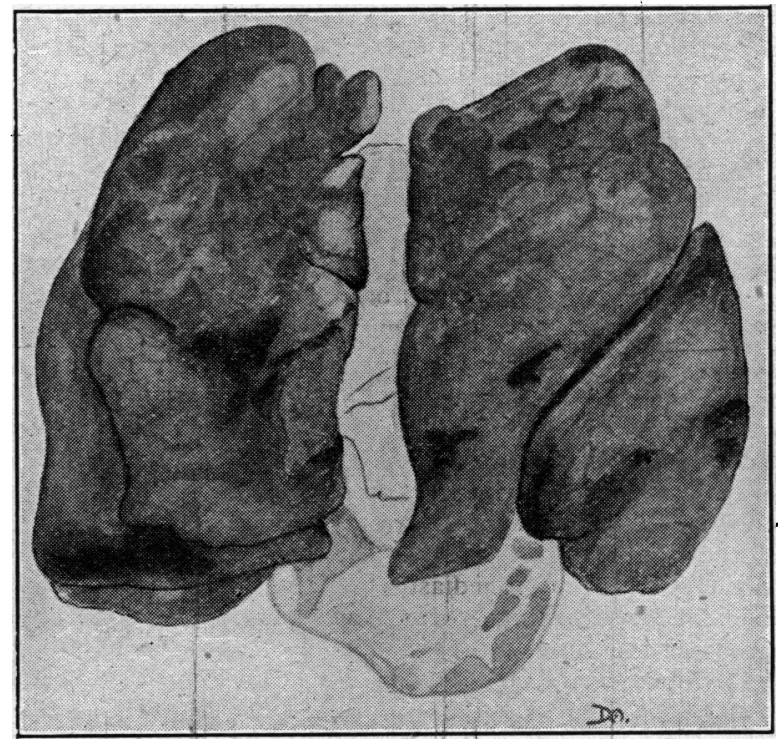

Typical appearance of a lung in cases of blast injury. Note the general congestion, with darker areas of haemorrhage. On the right lung these darker areas are located along the interlobar septa, and on the left they have taken on a linear distribution.

regard these, especially in older people, as corresponding to intercostal spaces rather than to ribs. Apart from these intercostal markings there is no special predilection for the areas of severe haemorrhage, but as a rule the regions most affected are those parts of the lung which expand indirectly-i.e., the posterior surface of the lung apex and the posterior surfaces of the lungs 
in contact with the spinal column and attached segments of the ribs-and laterally the maximum effect is in the middle partsi.e., opposite the 5th, 6th, and 7th ribs (as shown in the accompanying illustration). The picture is bilateral, though one lung may be affected more than the other. However, there is no general rule as to the position of the body in relation to the source of blast and the severity of the haemorrhage, since other factors operate. Apart from this picture of haemorrhage and emphysema the only other naked-eye appearance of significance is the presence of subpleural bullae. These occur in only a small proportion of cases, but are very definite. The pleural spaces usually contain blood-stained fluid.

The histological picture of those dying early is what one would expect from the macroscopic appearance. There is widespread congestion, often with intra-alveolar haemorrhage to a very marked extent. The alveoli may be ruptured and mild oedema may be present even in the early stages. There is no definite relation between the size of the haemorrhages and the survival period, but most of the bleeding would appear to take place almost at once. Later, fibrin formation and round-cell infiltration occur.

The after-course of these cases is gradually becoming clearer. That many are mild enough to be subclinical throughout their whole course is apparent from post-mortem studies of patients subjected to blast and dying later of a complicating lesion. The incidence of blast lesions of a minor degree among air-raid casualties would appear to be high. They seem to clear up in time with little symptomatology and no sequelae. The clinical cases pass through a stage of bleeding which is probably quite short (4 to 6 hours), then a stage of coagulation, absorption, and repair somewhat as occurs with a pneumonic exudate. Patients who die do so as a rule because of injuries elsewhere, but those subjected to pure blast seem either to die in the first few hours or to recover if no complication sets in. The course of these cases, however, is beset with three dangers: (1) development of pneumothorax; (2) acute oedema and heart failure; (3) infection-bronchopneumonia.

One of my patients developed a pneumothorax shortly after admission to hospital and died within 24 hours. A subpleural bulla which had ruptured was present on the surface of the right lower lobe. The histological picture of the lungs was characteristic of blast injury. He had received no direct chest injury. Cases such as the one described below would appear to be those most liable to develop acute oedema and heart failure.

A man aged 24 was standing at the entrance of a shelter when a bomb exploded $20 \mathrm{ft}$. away. The blast was considerable and a bomb splinter injured his foot. On admission to hospital be had lost a considerable amount of blood, was shocked, and as amputation of his foot was necessary a transfusion of whole blood was given. He died next day with clinical signs of right-sided heart failure. At necropsy were found dilatation of the heart and extensive pulmonary oedema superimposed on blast changes-a condition contirmed by histological examination.

Finally, since we are dealing with a lung which is full of haemorrhagic areas and with a patient whose vitality is lowered by shock and whose treatment demands a certain amount of immobility, we would expect infection to develop. On the whole, however, a pneumonic process was comparatively rare in this series.

An aspect of blast injuries which has received little attention in the literature is the mediastinal changes which occur. I have found contusion of the myocardium in several cases, and, in one, rupture of the aorta, while many have petechial and small haemorrhagic areas in the anterior mediastinum, all pointing to mediastinal trauma. It is important, therefore, for the physician to examine all cases of blast with respect to cardiac symptoms such as tachycardia, dilatation, and cardiac irregularity.

\section{Two Problems of Blast Injuries}

In the elucidation of blast injuries two problems present themselves: What is the mechanism of the production of the lung damage? and What is the cause of death? Two theories can explain the lung injuries-direct trauma to the chest wall, or a force acting down the trachea and bronchi, causing either a negative or a positive pressure wave. Direct trauma would seem to explain the condition much better than any other theory, and, indeed, is probably the only one which at the same time accounts for the lung damage and mediastinal injuries as well as the abdominal lesions found. It is probable that in pulmonary concussion we are not dealing with a new condition but with contusion of the lung caused by a fresh set of circumstances as a result of war. When a bomb explodes, the body is at once placed in a medium of high pressure such as that in which a diver would find himself if unprotected at a depth ; and so the abdominal and thoracic cavities, with their relatively weak walls, give way before the external pressure, and the underlying organs are damaged, especially elastic organs such as the lungs.

Four main theories have been advanced as to the cause of death in these cases. First, since the lungs appear to suffer most, many workers have thought that death might be due to the pulmonary haemorrhages and interference with respiratory function. Examination of blast cases shows that, while this might be true in some cases, the lung injury is usually not sufficient to cause death. Secondly, some have postulated a cerebral cause of death. The lesions in the brain in those dying immediately or soon after injury are very indefinite, but if we postulate a wave of positive pressure coming up from the abdomen and thorax via the blood stream and damaging the vital centres no gross pathological change would be expected. Thus, while positive evidence is scanty still, this theory is the most reasonable one yet formulated. Thirdly, death may be cardiac in origin. I have stressed the importance of injury to the heart in cases of blast, and there is quite a chance that ventricular fibrillation may occur and account for death on certain occasions. Fourthly, air embolism may be a possible cause of death. In blast the body is suddenly immersed in a wave of high velocity and pressure, the picture somewhat resembling that of a diver released unprotected at a depth. The gaseous content of blood and tissue fluids may thus be increased even though the effect of the blast wave is momentary. Then, as the effect of the blast wave passes off, the body is faced with an impossible situation, as in the case of a diver immediately brought to the surface, and air embolism is the result.

\section{Pulmonary Fat Embolism}

Reported cases of pulmonary fat embolism have rapidly increased in number in recent years owing to wider recognition of the condition. This is usually a sequel to fracture of a long bone, especially the femur, the fat gaining entrance to the blood stream and being carried to the lungs; thence it passes into the general circulation, causing pathological changes in the brain, kidneys, and heart muscle.

In a series of cases reported by Wilson and Salisbury in 1942 the main macroscopic lung findings were haemorrhage and mild oedema. The congestion and haemorrhage may be considerable, but I did not see the large subpleural haemorrhages described by Robb-Smith. These are much more characteristic of pulmonary concussion. Small areas of subpleural haemorrhage may be present. On cutting into the lung surface the picture is one of extreme congestion with small areas of haemorrhage. Microscopically, fat can be demonstrated in fresh preparations by Robb-Smith's technique, though I have found that staining the lung tissue with Wright's stain gives more convincing results. When stained with osmic acid, fat droplets can be seen in the capillaries. The alveoli contain red cells and oedematous fluid, the red cells being much less numerous than in blast cases, but the oedema is usually much more pronounced.

\section{Summary}

The main pathological features occurring in closed chest injuries are outlined.

In laceration the chief danger is haemothorax, the pathology of which is here dealt with.

The pathology of pulmonary contusion is compared with the effect of blast, and the evidence for regarding pulmonary concussion as being due to the direct trauma to the chest wall is outlined.

The importance of cardiac lesions in chest injury is stressed. Lung changes in cases of fat embolism are briefly discussed.

I wish to thank Col. A. S. Heale for permission to publish this paper. I am also grateful to Prof. Debono for his co-operation; to Prof. Bernard, Director of Public Health, and the authorities of the Central Civil Hospital for allowing me such free access to their wealth 
of material; to the staff of the General Hospital; and to Majors A. Charles and R. E. Tunbridge, R.A.M.C., for much help and criticism. Major D. J. S. Armstrong, R.A.M.C., supplied the drawing that is here reproduced.

\section{Biblography}

Alexander, J. Browning (1940). Post-grad. med. J., 16, 84.

Anderson, R. G. (1940). British 1 .
Barber, H. (1938). Ibid., 1, 433 .

Barber, (1940). Ibid., 2, 520 .

Bright, E. F., and Beck, C. S. (1935). Amer. Heart J., 10, 293

Edwards, F. R., and Davies, H. Morriston (1940). Lancet, 2, 673.

Fallon, M. (1940). Brit. J. Surg., 28, 39.

Hadfield, G. (1941). Proc. roy. Soc. Med., 34, 189.

- (1941). Lancet, $2,197$.

(1941). British Medical Journal, 2, 239.

and Christie, R. V. (1941) Ibid, 1, 77.

478.

Kahn, M. H., and Kahn, S. (1928). Ann. intern. Med., 2, 1013.

Robb-Smith, Ä. H. T. (1941). Lancet, i, 135.

(1941). Ibid., 2, 198.

- (1941). British Medical Journal, 2, 241.

Ross, Joan M. (1941). Jbid., 1, 79.

(1941). Ibid., 2, 241.

Scadding, J. G. (1941). British Medical Journal, 2, 57.

Sellors, T. Holmes (1941). Ibid., 1, 825

Warburg, E. (1938). Traumatic Heart Lesions, Humphrey Milford, London.

Zuckerman, S. (1940). I ancet, 2, 219

(1941). Proc. roy. Soc. Med., 34, 171.

\section{AN OUTBREAK OF EPIDEMIC CATARRHAL JAUNDICE}

BY

L. R. L. EDWARDS, M.R.C.S., L.R.C.P., D.P.H.

Senior Assistant Medical Officer of Health, County Borough of Wolverhampton

A review of accounts of outbreaks of epidemic catarrhal jaundice occurring in this country shows that the majority have affected rural areas. There have been exceptions, for Morgan and Brown in 1927 described an extensive outbreak in the Midlands in which 200 persons were affected, with one fatality. A previous outbreak in Staffordshire was described by Frazer in 1935, although Barber (1937) regarded this epidemic as one of infective hepatic jaundice. It would appear that the town-dweller has relative immunity from this disease. The following account of an outbreak of epidemic catarrhal jaundice occurring in an urban area may therefore be of some interest; it is published in the hope that other writers may suggest further aetiological factors and effective measures of control.

\section{Incidence}

A total of 64 cases occurred in an outbreak which has lasted for eight months. This figure is estimated to represent about $80 \%$ of the total number of cases. The outbreak was chiefly among school-children, and the few adults affected were mainly young school-teachers. The epidemic appeared to be confined to one school for several months, but after a summer holiday, during which only a limited number of children attended school, the disease made its appearance at several other schools. Practically all of these schools were in the northern part of the borough.

The seasonal incidence of the disease is usually in autumn and winter, but this epidemic began in the spring and continued over the summer. The sex incidence was about equal. The highest age incidence occurred between 6 and 10 and between 20 and 30 years. It was noted that in no case were more than two members of a family affected. School-teachers were particularly at risk to the disease.

\begin{tabular}{|c|c|c|c|c|c|}
\hline \multicolumn{4}{|c|}{ Age } & \multirow{2}{*}{$\begin{array}{c}\text { Males } \\
5 \\
20 \\
3 \\
\text { Nil } \\
1\end{array}$} & \multirow{2}{*}{$\begin{array}{c}\text { Females } \\
3 \\
22 \\
3 \\
5 \text { (4 school-teachers) } \\
2 \text { (1 school-teacher) }\end{array}$} \\
\hline $\begin{array}{c}4-5 \text { yea } \\
6-10 " \\
11-15 " \\
20-30 " \\
\text { Over } 30 "\end{array}$ & $\begin{array}{l}\text { inc. } \\
" \\
"\end{array}$ & $\begin{array}{l}\because \\
\because \\
\because\end{array}$ & $\begin{array}{l}\ddot{0} \\
\ddot{0} \\
\ddot{x}\end{array}$ & & \\
\hline \multicolumn{2}{|c|}{ Total } & .. & .. & 29 & 35 \\
\hline
\end{tabular}

\section{Aetiology}

The evidence points to a contact at school. The epidemic had not the explosive nature usually associated with outbreaks of food-poisoning due to contaminated food, milk, or water. Further, the water supplies of the borough are chlorinated, and school milk supplies are bacteriologically clean. Again, the infant and junior departments in the school mainly affected received milk supplies from different sources. The disease appeared to pick out the children in twos and threes from each class, and the available evidence pointed to a close personal contact, with droplet infection as the probable means of spread. No bacteriological examinations were carried out, but reports of previous epidemics show that they are negative and that the probable cause of the disease is a filterable and ultramicroscopic virus. There was no particular incidence of sore throats or enteritis. While an epidemic of enteritis occurred in some of the schools of the borough, this was seasonal, and, further, there was no especial incidence of cases of catarrhal jaundice at any of them.

\section{Clinical History}

From descriptions given by various medical practitioners, and from visits made to many of the cases, the following clinical report has been compiled.

The spread of the disease suggests that the incubation period varies between three and four weeks. In the prodromal period malaise and anorexia may be present, but more often the disease started with vomiting, sometimes associated with severe upper abdominal pain. The attack of vomiting lasted from a few days up to a week, and resembled cyclical vomiting. Constipation was usual, though a few cases had diarrhoea until the onset of jaundice. In some cases jaundice limited to an icteric tinge of the conjunctiva, and nausea, were the only symptoms, and the illness cleared up in less than a week. With the cessation of vomiting, jaundice appeared first of all in the conjunctiva, and in the course of a day or two spread to the skin of the face, trunk, arms, and lower limbs. The colour deepened to a canary yellow, and the motions became claycoloured and the urine orange-coloured. Pyrexia was variable in occurrence and in severity. Headache was sometimes severe. There was well-marked enlargement of the liver, with tenderness over the gall-bladder. The spleen was enlarged. The jaundice lasted a variable period from a few days up to two weeks, and gradually disappeared in the reverse order. The faeces and urine became normal a few days before the jaundice disappeared from the skin.

Examination of blood films from five of the cases in the acute stage showed a well-marked leucopenia with a relative lymphocytosis. An increase in monocytes was noted in each film. In the acute stage in adults there was sometimes complaint of severe pains in back, arms, and legs. No epistaxis or petechial rashes occurred. Despite the severe jaundice, itching of the skin was present in very few cases. Convalescence was marked by the development of excessive appetite. In a few instances some degree of furunculosis occurred.

Vomiting in the acute stage is usually a feature; Lisney (1937) attributes this to duodenitis. According to Pickles (1930), the severity of the upper abdominal pain may suggest a perforation.

\section{Differential Diagnosis}

The diseases to be excluded are Weil's disease, infective hepatic jaundice, simple catarrhal jaundice, enteric fevers, and acute yellow atrophy. Weil's disease is marked by epistaxis and petechial rashes, with high fever, severe nervous disturbance, blood leucocytosis, and the identification of leptospira in the blood. Infective hepatic jaundice is characterized by an absence of pre-icteric symptoms, and the jaundice is less marked. Simple catarrhal jaundice occurs sporadically. The symptoms are very similar to those of epidemic catarrhal jaundice, and this complaint may be an interepidemic type. Jaundice seldom occurs in the enteric group, while acute yellow atrophy is a very rare disease.

\section{Spread of the Disease}

The spread of the outbreak is well illustrated by the experience of the junior department of one of the schools.

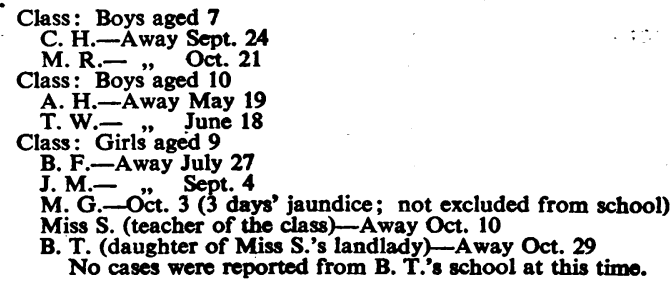

